\title{
En el corazón mismo de la colonialidad. Poder, totalidad social y heterogeneidad en las primeras obras sociológicas de Aníbal Quijano (1962-1966) ${ }^{1}$
}

Recibido: 10/03/2019

Aprobado: 24/05/2019

\author{
DANILO ASSIS CLÍMACO \\ Universidad Nacional Mayor de San Marcos \\ daniloclimaco@yahoo.com.br \\ YURI GÓMEZ \\ Pontificia Universidad Católica del Perú \\ yuquios01@gmail.com
}

\begin{abstract}
RESUMEN
La capacidad que tuvo Aníbal Quijano de mantenerse como un autor referencial por más de cuatro décadas en el debate latinoamericano no se debía a un estilo camaleónico, sino más bien a un conjunto de ejes significativamente sólidos desde los cuales percibía e indagaba las transformaciones de lo social y de los debates intelectuales. El texto busca mostrar que algunos de los términos que habitan en lo que Quijano cierta vez denominó como "el corazón mismo epistémico de la idea de colonialidad del poder" (2013): poder, totalidad social y heterogeneidad, se encuentran ya en sus primeros trabajos, constituyendo una forma de indagar la realidad sumamente fértil.
\end{abstract}

Palabras clave: Poder, totalidad social, heterogeneidad.

\section{At the heart itself of coloniality. Power, social totality and heterogeneity in the fisrt sociological works of Anibal Quijano (1962-1966)}

\begin{abstract}
The capacity of Anibal Quijano to remain as an influential author in the Latin American debate for over four decades does not obey a chameleonic style. Rather than a number of fundamentally solid axes from which he perceived and explored social transformations and intellectual debates. The article seeks to reveal some of the concepts that inhabit what Quijano once called "the epistemic heart itself of the idea of coloniality of power" (2013): power, social totality, and heterogeneity, these are already present in his first works. Thus becoming an extremely fertile method for exploring reality.
\end{abstract}

Keywords: Power, social totality, heterogeneity

1 El texto ha sido enriquecido por los comentarios de Manuel Valladares Quijano, Amarildo Figueroa y Antonio Romero. Agradecemos enormemente sus amables lecturas. 


\section{La necesidad de un mínimo biográfico, a modo de introducción}

Como ya ha sido observado, el enorme reconocimiento a Aníbal Quijano por la teorización de la colonialidad del poder no ha venido acompañado de un debate sobre el conjunto de su obra, lo que supone límites importantes para comprender su pensamiento, el contexto histórico y político en que emergió, $\mathrm{y}$, sobre todo, el modo como este contexto se relaciona con el contemporáneo (Assis Clímaco, 2014; Segato, 2013). Una adopción de la colonialidad del poder que no observe la continuidad de la obra del autor implica, en última instancia, desconocer la historicidad del propio patrón de poder colonial/ capitalista.

La noción de heterogeneidad histórico-estructural ha sido apuntada por el propio Aníbal en diferentes ocasiones ${ }^{2}$ como compartiendo el "mismo corazón" que la teoría de la colonialidad del poder. Si bien es en el archiconocido "Colonialidad del poder y clasificación social" (2014[2000]) que la teorización más madura de este concepto ocurre, es indudable que los elementos que lo componen - la perspectiva de una totalidad social analíticamente divisible en diferentes ámbitos y compuesta por tiempos históricos heterogéneos articulados por un patrón de poder que dan forma, es decir, estructuran los conflictos entre los diferentes grupos sociales - no solo se encuentran muy temprano en su obra, sino que parecen conformar la expresión mayor de su sensibilidad intelectual.

Aníbal nace en 1930, en el seno de una familia comprometida con una transformación social que era palpable en las conquistas paulatinas que venían logrando los pueblos en el Perú. La vida intelectual en provincia se consideraba, y efectivamente parecía ser (Quijano, 2016), más audaz que la limeña, con vívidos debates sobre horizontes sociales libres de la jerarquía y la violencia coloniales. Amante de la literatura y la poesía, era dotado de una curiosidad irrestricta, siendo Medicina su primera aspiración profesional. Así, viene a Lima en 1947 a matricularse en los estudios generales de ciencias de la Universidad Nacional Mayor de San Marcos. Aprista desde los catorce años, se torna en Lima uno de los más importantes líderes estudiantiles. La dictadura lo llevaría a la cárcel este año. Los debates políticos en este espacio, unido a la decepción con la dirección del APRA, que se negó a radicalizarse

$2 \quad$ Notablemente en el 2013. 
como demandaban muchas de sus bases, lo aproxima al marxismo y también a las ciencias sociales. ${ }^{3}$

En 1948 se cambió a Estudios Generales de Letras y la amplitud de sus intereses lo condujo a recorrer una vasta variedad de materias en tres carreras distintas: Letras, Derecho e Historia. Pero la disciplina que abrazará como suya es la sociología, aún incipiente en el Perú, por cuya autonomía académica él vendría a luchar en la década de 1960. En tanto en su interpretación, era la ciencia responsable por las tendencias generales de la sociedad, Quijano consideraba la sociología como esencial para comprender e influir en los procesos de transformación más profundos de la sociedad.

En búsqueda de esta sensibilidad intelectual, en lo sucesivo abordaremos cómo Quijano piensa estas cuestiones en sus primeros artículos académicos, publicados entre 1962 y 1966. Para mayor claridad tanto para quienes escribimos como para quienes leen el texto, los hemos subdivididos en tres categorías diferentes. En la primera se encuentra exclusivamente un texto sobre las luchas revolucionarias en el Perú, en donde Quijano expresa de manera límpida el horizonte político que guiaba su obra. En la segunda están tres textos predominantemente teóricos, que nos ofrecen su interpretación de las ciencias sociales, la sociología en particular. Finalmente, los textos sobre las tendencias sociales en el Perú y en América Latina - el grupo cholo, la relación urbano-rural, los movimientos campesinos y la marginalidad-, en los que encontramos cómo, en relación a sus horizontes teórico y político, nuestro autor indaga la realidad. Por estos años publicó "La poesía: una praxis", texto que se encuentra en nuestro horizonte de análisis, pero sin que lo hayamos expuesto a una lectura intensa, dado que no aborda — de forma directa - las preocupaciones sociológicas del autor.

\section{Las bases políticas: un periodo revolucionario}

"En la última década, Latinoamérica ha ingresado, definitivamente, en un periodo revolucionario" (Quijano, 1971a[1966], p. 15), es la primera frase de un texto de coyuntura política, "Las experiencias de la última etapa de las luchas revolucionarias en el Perú”. Frente a tan grave convicción, cabía asumir una

3 Como recuerda Manuel Valladares (comunicación personal), Quijano se abstuvo de hacer públicas sus diferencias con el APRA para no contribuir a la fragilización del partido, cuyos integrantes se encontraban también perseguidos y en riesgo de vida. 
responsabilidad equivalente, lo que para Quijano (1971b, p. 15) implicaba una actuación informada tanto por las especificidades de cada país, como por el carácter mundial de la lucha de clases:

El proceso envuelve a la totalidad de los países y nacionalidades de la región; pero se desarrolla en diversas formas y en diversos niveles, según las diferentes circunstancias históricas de cada país. Si se quiere llegar a la necesaria integración sistemática de los diversos procesos particulares, en el curso del enfrentamiento global inevitable con el imperialismo, es indispensable tratar de comprender y evaluar constantemente cada uno de ellos y captar su lugar y su significado para la problemática general del proceso revolucionario en Latinoamérica.

El texto fue escrito para la revista marxista chilena Estrategia, lo que liberó a Quijano de cubrir expectativas científicas neutrales a las que estaban expuestos textos suyos publicados en otros medios. Esto permitió expresar la postura política de Quijano, su expectativa en una lucha revolucionaria que pasara también por la experiencia de las armas, y que se encontraba expuesta de una forma excepcionalmente clara.

Quijano sustenta que el periodo se encuentra marcado por un desplazamiento de la lucha revolucionaria de la ciudad hacia el campo, lo cual habría sido fruto de dos grandes acontecimientos ocurridos a finales de la década de 1950, uno interno: la consolidación y rápida expansión de las luchas campesinas por la recuperación de tierra, y otro externo: la Revolución cubana, que despertó gran entusiasmo entre sectores urbanos que pasarían a organizar grupos guerrilleros.

Las luchas campesinas eran conformadas por una notoria interacción entre diferentes grupos culturales. Por un lado, el proceso de sindicalización había sido iniciado por un peruano de origen criollo (occidental/urbano) y con formación trotskista en Argentina: Hugo Blanco, quien desde 1957 organizó la lucha por la recuperación de tierras en los valles de La Convención y de Lares en Cusco. El éxito de la experiencia incentivó nuevos desplazamientos de grupos urbanos de izquierda hacia el campo, pero sobre todo llamó la atención de las comunidades indígenas - que Quijano considera son cholas antes que indígenas, porque sus integrantes atravesaban por un proceso de integración de elementos occidentales e indígenas en los diferentes ámbitos de su vida-, las cuales han expandido sin un liderazgo externo la toma de tierras e incluso el establecimiento de formas de ejercicio de poder propias. 
La guerrilla venía organizándose desde inicios de la década de 1960, pero la Revolución cubana era tomada "como modelo y no como punto de partida" (1971b, p. 54), lo que implicaba un desdén por el contexto peruano, por lo cual se desestimaba una aproximación a las comunidades campesinas de las zonas en que se establecían las guerrillas. De esta forma, los primeros grupos guerrilleros, frente a la violencia desplegada tras el golpe de Estado de 1962, no lograron defenderse y la mayoría de sus integrantes terminaron muertos o encarcelados. Paralelamente, una represión equivalente hacia sindicatos y comunidades que realizaban tomas de tierra provocaron masacres y encarcelamientos, incluido el de Hugo Blanco.

Quijano observa que la ausencia de un sistemático examen sobre la realidad del país facilitó el trabajo represivo, pero también la propia izquierda parecía empezar a darse cuenta de ello. Para diferentes tendencias se había hecho obvio que la guerrilla no habría tenido derrotas tan fuertes si hubiera contado con un respaldo popular comunal, a la vez que la represión a las tomas de tierras hubiera sido menor si contaran con una defensa armada. En este sentido, el desplazamiento de las próximas experiencias hacia el sur andino, con una década de experiencias en toma de tierras, era un paso visto como necesario para diferentes actores. Ya los grupos revolucionarios urbanos, que desde hace algunos años estaban migrando hacia el campo, iniciaron un proceso de mayor integración entre sí y con los grupos campesinos, aunque Quijano observa un importante déficit en la construcción de un movimiento obrero revolucionario. ${ }^{4}$

Para los objetivos de este artículo, nos gustaría resaltar dos aspectos. En primer lugar, el horizonte político revolucionario en el cual se inscribe todo el pensamiento de Quijano. En segundo lugar, el modo como su preocupación política exige un conocimiento al mismo tiempo atento a la totalidad de lo social — que aquí se refiere al periodo de transformación en toda América Latina, frente a la hegemonía imperialista - como a las especificidades de cada uno de sus componentes, que el texto pone de manifiesto al detenerse a

4 En estos años, sus textos se detienen poco o nada en el análisis de las luchas urbanas. Sabemos por testimonios del autor y de personas próximas que entre las décadas de 1940 y 1960, además de haber sido un líder estudiantil muy reconocido, Quijano apoyaba distintos sindicatos y participaba de numerosos espacios políticos de izquierda, conviviendo asimismo con presos políticos de las más diferentes tendencias en los periodos en que estuvo encarcelado. Debemos suponer que la efervescencia de la lucha campesina, por su emergencia relativamente súbita y por la radicalidad alcanzada — superando, incluso, los límites que Marx había observado en el campesinado (Quijano, 1979b[1965])—, lo impresionaron a punto de limitarle, por un periodo, de reflexionar sobre las luchas urbanas, las cuales serán su objeto preferente de acción teórica y política desde que regresa de Chile al Perú en 1971. 
detallar las diferentes características de cada uno de los grupos en lucha en ese momento, además de buscar explícitamente contribuir con los esfuerzos de quienes en estos grupos venían dándose cuenta de que la experiencia cubana no era exportable, sino adaptable.

\title{
3. Las bases teóricas: una sociología de oposición desde Perú y América Latina
}

\begin{abstract}
El valor de las ciencias de la sociedad [...] consiste en la posibilidad de predecir los fenómenos sociales, permitiendo al hombre intervenir de manera racional y consciente, es decir, libre, en la organización de las condiciones de su propia existencia (Quijano, 1971b[1965]).
\end{abstract}

Aun cuando su estima por el $\operatorname{arte}^{5}$ y por las fuerzas de la vida comunitaria y cotidiana eran enormes, Quijano consideraba que las ciencias sociales, especialmente la sociología, tenían un alcance de conocimiento sobre lo social de mayor profundidad y alcanzable por otros ámbitos en que la sociedad también se pensaba a sí misma. Tal prominencia se relacionaba a la consideración de la sociología como necesariamente crítica, desveladora de los aspectos estructurales de la sociedad encubiertos por los grupos de poder. La perspectiva hegemónica en la sociología, el estructural-funcionalismo, era denunciada en tanto sus pretensiones de objetividad plena y su comprensión armónica y ahistórica de lo social participaban de los mecanismos de camuflaje de los conflictos sociales requerido por las élites.

Deslindándose también de las perspectivas meramente idealistas o marcadamente especulativas, apuesta por una ciencia social que relaciona dialécticamente lo teórico y lo empírico,${ }^{6}$ con la finalidad de "descubrir, entre el fárrago de los hechos históricos menudos, las regularidades grandes que presiden la existencia y continuidad de las sociedades en el curso del tiempo" (Quijano, 1964b, p. 55). Este carácter predictivo de la sociología es lo que habilitaría a la sociología a ser un fundamento imprescindible para que "el

$5 \quad$ "El poeta se revela a sí mismo, entrega su propia y desnuda presencia, su propio modo de participar en la realidad, su modo de ceder a ella o de transformarla, las condiciones y las circunstancias de su propia lucha. Porque la poesía es un modo de relación entre el hombre y su realidad, el más directo, el más intenso, el más profundo. El más hermoso" (Quijano, 1964a, pp. 1-2).

6 "La ciencia sociológica contemporánea se caracteriza, precisamente, por la permanente e insustituible interdependencia dialéctica entre la teoría, que guía y orienta la búsqueda y organización de los datos concretos, y empírica, y los resultados de esta labor que permiten modificar, ampliar profundizar e innovar, en general, constantemente la teoría" (1971b, p. 11). 
hombre" interviniera de forma consciente en la construcción de su realidad, lo que parece ser para nuestro autor un sinónimo de libertad.

A las suspicacias frente a una sociología neutra, Quijano añade otra advertencia: las ciencias sociales de las sociedades industrializadas, aun siendo críticas, son realizadas no solo en sociedades por demás diferentes, sino frente a las cuales las nuestras se encuentran en situación de dependencia; por tanto, la adopción de sus teorías puede obviar elementos centrales para develar las formas de dominación que se sufren en Latinoamérica, redundando en un etnocentrismo y en "la prolongación de un colonialismo cultural" (1971b, p. 14). Ello implicaba precaución, antes que restricción, a su lectura de autores europeos y estadounidenses. Una muestra de ello es su ensayo sobre Saint-Simon y sus discípulos, cuyas ideas parecen coincidir con las formas de conocimiento que Quijano (1964b, p. 50) aspiraba formular o encontrar:

Parece ser que es por primera vez en la historia del pensamiento occidental que aparece con Saint-Simon la idea de sociedad como una totalidad cambiante, cuyos elementos formativos están en estrecha interdependencia entre sí y con el conjunto orgánicamente estructurado.

En oposición al estructural-funcionalismo que interpreta la sociedad como un ente estático, sustentado en la funcionalidad que cada uno de sus elementos cumple para el conjunto, aquí hay una noción de interdependencia abierta, es decir, permeable a la reconfiguración de la relación entre sus elementos y de este con el todo. Además, nuestro autor - recordemos, historiador de formación - rescata de Saint-Simon "la radical historicidad de todos los fenómenos sociales" (Quijano, 1964b, pp. 54-55), lo que refiere a la inestabilidad inherente a la totalidad social derivada de la necesaria divergencia de fuerzas entre los diferentes intereses que la componen. La conflictividad es una marca de lo social y el motor de cambios que no pueden ser relativos a solo una parte de ella, sino a su totalidad, dado que todos sus grupos y sectores se encuentran estructurados conjuntamente. Así, Saint-Simon se soslayaba de tendencias de pensamiento social que fragmentaban las relaciones, dentro de una percepción societal que confluía con los intereses de los grupos dominantes.

La importancia de lo histórico radicaba, asimismo, en el futuro: la conciencia de ser parte de colectividades expuestas al tiempo permitió a la generación de Saint-Simon y a las posteriores considerarse como partícipes de la construcción de la vida colectiva: "La sociedad es cómo un órgano que genera órganos" (Quijano, 1964b, p. 52). Esta asociación entre lo racional y la cons- 
trucción de un sí mismo colectivo es lo que permite la asociación de libertad y razón en un mismo horizonte histórico. En todo caso, ello conlleva a que las ciencias sociales sean en última instancia "ciencias de oposición” (Quijano, 1971b), necesariamente reñidas con la necesidad de las élites de impedir una racionalización de lo social.

La historicidad, en consecuencia, se deslinda de la tradición especulativa predominante en la sociología peruana, denunciada en "imagen y tarea de la sociología peruana" (1971b[1965]). Al mismo tiempo, la centralidad de este criterio reclama por un conocimiento contextualizado. Quijano será reiterativo sobre esta cuestión. La sociología como disciplina no equivale a la reproducción automática de las premisas que operan en la sociedad donde tuvo su origen. Esto adquiere relevancia cuando el motor de la sociedad a conocer es el cambio constante, en donde la contradicción y el conflicto emergen de la naturaleza propia de la sociedad latinoamericana, muy distinta a los procesos embrionarios de las sociedades industriales europeas. En ese sentido, toda nueva formación social surge o tiene un desarrollo embrionario en la formación social precedente, esta mirada será fundamental para una comprensión de lo histórico como transformación.

\section{Los estudios sobre las tendencias de la sociedad latinoamericana: la relación urbano-rural, lo cholo y la marginalidad en la situación de dependencia}

Los cinco textos de Quijano del periodo considerado, "Lo cholo y el conflicto cultural en el Perú" (1980[1964]), "El movimiento campesino en el Perú y sus líderes" (1979b[1965]), "Los movimientos campesinos contemporáneos en América Latina” (1979a[1966]), “Aspectos socioculturales de la urbanización en América Latina" (1977a[1966]) y "Notas sobre el concepto de 'marginalidad social'" (1977b[1966]), nos muestran un conjunto de cuestiones interrelacionadas - casi como si se tratara de una sola gran investigación, cuyos resultados parciales van siendo expuestos según su grado de maduración — que buscan dar cuenta de los rasgos fundamentales del extraordinario periodo de transformaciones que vivía el mundo y América Latina desde la década de 1930.

Las tendencias mayores que se desprenden de estos textos se relacionan a los procesos imbricados de industrialización, modernización y urbaniza- 
ción, y a la radicalización de las luchas sociales, sobre todo campesinas. Desde su posición subordinada en el "sistema internacional de dependencia que caracteriza al sistema capitalista mundial” (Quijano, 1980[1964], p. 55), Latinoamérica cumplió en treinta años un proceso que Europa hizo en trescientos: la superación a nivel poblacional de lo urbano sobre lo rural, espacio donde este pasa a ser dependiente de aquel por la preponderancia que los productos manufacturados logran sobre los primarios y, aún más, por un imaginario moderno que proyecta la vida cultural y material urbana como el horizonte de mayor satisfacción vital.

Otrosí, nuestros espacios urbanos carecen de infraestructura e interés político para absorber las poblaciones que llegan a los centros urbanos de mayor modernización en cada país. Sistemas previamente inconcebibles de transporte y comunicaciones - construidos principalmente desde la lógica de enclave, según interés imperialistas - tienen como efecto colateral la aproximación entre sí de diferentes regiones de cada país, atizando la movilidad y la comunicación de las poblaciones, las cuales pasan a ser cada vez más inclasificables en los antiguos términos de rural y urbano. Se produce asimismo una heterogeneización en todos los ámbitos de la vida social, surgiendo grupos sociales que no se adscriben a una sola identidad o lo hacen a identidades sociológicamente nuevas, como la del cholo. Emerge asimismo una población denominada marginal que abandona ciudades no modernizadas o el campo en dirección a los grandes centros sin condiciones de posicionarse en estos de forma plena, mientras en el campo los intentos de recuperación de tierra por parte de los pueblos — sobre todo, pero no solamente, indígenas - logra avanzos notables, dada la debilidad relativa de las élites rurales frente a la súbita urbanización.

Desde este escenario amplio, Quijano va descendiendo hasta los fenómenos más concretos, haciendo pausas en los niveles intermedios. Tomando como ejemplo a "Los movimientos campesinos contemporáneos en Latinoamérica" (Quijano, 1979a[1965]), tenemos a nivel macro, por un lado, que el inédito vigor de las luchas campesinas se relaciona al proceso de debilitamiento de las élites rurales tradicionales frente a élites burguesas o moderno-rurales, lo cual a su vez deriva del nuevo posicionamiento de Latinoamérica frente a la ampliación regular pero no sin inconsistencias del proceso imperialista en la región. Por otro lado, la ampliación de redes viales e informacionales, junto con la radicalización y ampliación de los grupos de izquierda urbanos, permitieron una mayor extensión de las redes de alianza de los campesinos entre 
sí y junto con grupos urbanos, lo que en su conjunto les permitió alcanzar un carácter de clase - es decir, de conciencia de su especificidad socioeconómica- que Marx no había observado en los campesinos europeos y que tampoco parecía observable en los de América hasta recientemente. En un nivel intermediario, Quijano expone, dentro de los límites de una bibliografía que reconoce como insuficiente, las formas más relevantes de organización social campesina a través de dos etapas históricas, la "pre-política" — término utilizado solo en este texto-— y la "de politización". En la primera se encuentran los movimientos que denomina de "mesiánicos", "racistas", "agraristas tradicionales" y "bandolerismo social", los cuales son expuestos dentro de las especificidades histórico-sociales que le permitieron su emergencia en cada uno de los países en que se presentan. En su conjunto, se caracterizan por encontrarse más restrictos a objetivos concretos que no ponen en cuestión de forma amplia "los aspectos básicos de la estructura de poder social" (Quijano, 1979a, p. 58). El hacer frente a esta estructura es lo que justamente caracterizan los movimientos contemporáneos — que él categoriza como "agrarismo reformista", "bandolerismo político" y "agrarismo revolucionario"- . A partir de estas grandes tendencias, pasa a observar casos concretos, sobre todo los relativos al Perú. Además, de forma semejante a como ocurre en otros de sus escritos, el texto abre espacio a cuestiones de especial importancia para el autor, como por ejemplo, sobre el carácter de clase del campesinado contemporáneo —oponiéndose a tendencias de la izquierda que se niegan a reconocer este rasgo- , las formas de interacción entre los movimientos o la cuestión del liderazgo y su relación con las bases — Quijano observa que el liderazgo inicial, de origen urbano o cholo, no presupone una determinación externa sobre las bases, sino que son estas quienes imprimen radicalidad al proceso, reinventando según las circunstancias su modo organizativo, casi siempre rumbo a la consecución de sus objetivos, con menor temor que sus líderes al incremento de los conflictos con los terratenientes.

"Aspectos socioculturales de la urbanización en América Latina" (1977a[1966]) tiene una estructura similar. Nuevamente, desde la especificidad de la temática tratada, son presentadas las tendencias más generales del modo como América Latina se relaciona al sistema de interdependencia

7 Silvia Rivera afirma erróneamente, en un muy difundido escrito, que Quijano habría utilizado este término en 1983. Pero no vuelve a utilizar el término después de 1965. Este error de la socióloga boliviana no supone que su crítica estuviera mal encaminada. Efectivamente, por un lapso de algo más de diez años, Quijano se adhirió a presupuestos marxistas más rígidos que los que cultivó en la década de 1960. 
capitalista. Aquí Quijano advierte la existencia de un vasto sistema de sociedades y culturas interconectadas, asumiendo la existencia efectiva de un predominio de la cultura urbana de origen occidental sobre las demás, dado que la industrialización de la producción cultural y la masificación de los medios de comunicación y difusión garantizan su mayor capacidad para controlar los términos del intercambio de influencias culturales. De ahí que resultara necesario hablar de una dependencia cultural, no sin relación, pero con estatus propio frente a la dependencia económica y política. ${ }^{8}$ Aun así, para Quijano era también importante cuestionar las teorizaciones que abogaran por lo que denomina "planetarización" de la cultura, puesto que observaba por doquier que los pueblos también rechazaban o incorporaban de forma subversiva los términos culturales difundidos por lo urbano occidental, llegando a afirmar que "la emergencia de grupos socioculturales como el cholo en Perú y en otros países del área andina, o grupos equivalentes en otros países, representa una posibilidad sociocultural no colonial" (Quijano, 1977a[1966], p. 41). De esta forma, aun trabajando en los niveles más generales de las tendencias sociales, se resistía a considerar que estas tenían direccionamientos unívocos, sino que se encontraban mediatizados por especificidades históricas.

Los textos específicos que Quijano escribió sobre el Perú, "Lo cholo y el conflicto cultural en el Perú" (1980[1964]) y "El movimiento campesino en el Perú y sus líderes" (1979b[1965]), también parten de la comprensión de la inserción del país y el continente en el sistema de interdependencia capitalista, pero sus análisis se direccionan de forma más ágil hacia las problemáticas nacionales. El primer texto, probablemente el más conocido del autor en nuestro país, trae la consabida afirmación de que el Perú es una sociedad no en transición sino de transición, con lo cual llama la atención a que el sentido del proceso de cambio social en el país no tiene un sentido determinado, sino que depende de disputas que proporcionan una forma social relativamente inestable, inacabada, pero con un grado de estructuración social que le garantiza un status propio. La riqueza del texto deriva en larga medida del cuidado del autor en exponer cómo se manifiesta la vivencia del grupo cholo en los más diferentes ámbitos de la vida social, lo que a su vez lleva al autor a una comprensión de lo social que, aun bajo diferentes ropajes, lo acompañará toda la

8 Lo que distinguía el pensamiento de Aníbal Quijano de los teóricos de la dependencia que le eran contemporáneos, como recientemente ha recordado José Bengoa (2018), es su preocupación por la vida en su totalidad, no dejando que lo económico y lo política obliteraran lo ecológico, lo demográfico y, sobre todo, lo cultural. El cultivo de esta percepción de la totalidad le otorgaba una fina sensibilidad para comprender más que sus contemporáneos el modo como la potencia de la lucha se urdía en la vida cotidiana. 
vida, aquella que supone una composición de la estructura social por diferentes aspectos básicos, que entonces denomina como económicos, sociales, culturales, demográficos, psicológico-sociales y políticos (Quijano, 1980, p. 81).

Ya el texto sobre el movimiento campesino, muy en consonancia con los textos expuestos sobre la guerrilla en el Perú y sobre los movimientos campesinos en América Latina, ahonda en la especificidad de la experiencia en nuestro país. Reconociendo la enormidad de un proceso que en menos de diez años, y pese a masacres y encarcelamiento de líderes, seguía ampliándose a punto de alcanzar casi toda la sierra y parte de la costa y la ceja de selva, Quijano se dispone a exponernos sus rasgos centrales. Entre estos podríamos destacar que los alcances del movimiento amenazan la estructura de dominación completa del país, que participan de él toda la población rural - jornaleros, siervos, minifundiarios, integrantes de comunidades indígenas, asalariados, etc.--; que su liderazgo es eminentemente de origen urbano, criollo o cholo, pero que las bases son centrales en su radicalización; o que su capacidad política es inédita porque produce tanto Gobiernos locales propios como logra articulaciones cada vez más sólidas a nivel nacional.

El texto sobre marginalidad se distancia de los demás porque, pese a tratar una temática específica - incluso inédita, puesto que la migración de una población de origen rural hacia ciudades incapaces de incorporarlas en su seno era un fenómeno aún sin registro a nivel mundial-, la misma es abordada sobre todo desde una perspectiva teórica, buscando encontrar un camino de reflexión pertinente en un debate teórico amplio, pero de dudosos logros. Consecuente con su comprensión de lo social como una totalidad, buscaba una alternativa a la concepción de que las poblaciones marginales eran externas al ambiente social en el cual buscaban integrarse:

La marginalidad no puede consistir en una falta genérica de integración a la sociedad. Todo elemento marginal, aislado o en configuración con otros existe en la sociedad y porque existe esa sociedad. Forma parte de ella. El problema es que no forma parte de los patrones y tendencias que rigen sus estructuras dominantes, pero existe en dependencia de ella.

\section{Consideraciones finales}

Nuestro primer texto mostró sin ambages el objetivo político de Quijano: contribuir a la transformación revolucionaria de la sociedad, dando fin no 
solo al imperialismo, sino al sistema de dominación global. Buscaba evidenciar asimismo que el estudio minucioso de la realidad social en su conjunto - atendiendo a sus procesos de cambio tanto generales (en su nivel nacional, latinoamericano y mundial) como particulares (a nivel de las prácticas de lucha comunitarias y partidarias) - no es un preciosismo intelectual, sino una necesidad absoluta, y por ignorarla la izquierda peruana tuvo que pagar con vidas humanas y con la pérdida del control de haciendas reconquistadas. En este primer texto nos son dados ya, por lo tanto, una comprensión del poder (referido sobre todo mediante el término dominación), una comprensión de la totalidad social (dada por el periodo de luchas en el campo de América Latina) $\mathrm{y}$, finalmente, de la heterogeneidad (relativa a las especificidades históricas), la cual se relaciona de forma directa a los cambios sociales, entendidos como resultado de las luchas entre intereses contrapuestos.

Los textos teóricos afianzan el valor de las ciencias sociales en su carácter de oposición al orden establecido, el cual es desvelado mediante una relación dialéctica que va y viene entre una teorización abstracta, atenta a las tendencias más generales del orden social, e investigaciones empíricas rigurosas. Asimismo, es importante su rescate de la definición de sociedad de SaintSimon, pues al categorizarla como "totalidad cambiante", busca explicitar a la vez el modo como todos sus elementos están asociados entre sí, como su carácter histórico, transformador, resultado justamente de los intereses sociales diferenciados entre los grupos.

Los estudios sobre las tendencias sociales, finalmente, nos permiten percibir cómo Quijano vinculaba el carácter de abstracción máxima sociológica con la realidad concreta que requería la comprensión para ser, revolucionariamente, transformada. Aunque los cinco textos se encuentran entrelazados, no tienen una perspectiva totalmente unificada, la realidad — en su totalidad y en sus particularidades - presentada en cada uno son como trozos de un cristal roto que nos toca reconstituir. Para él, la América Latina de la década de 1960 vive un proceso acelerado de cambios sociales, asociados a los impactos de una súbita industrialización que tornó urbano a nuestros países, debilitando a las élites, permitiendo la emergencia y consolidación de luchas populares intensas y progresivamente más articuladas, sobre todo en el campo, donde la desestructuración de las relaciones favoreció fuertemente a las clases dominadas. Las luchas en las ciudades, sin embargo, eran aún relativamente débiles. Quijano notaba en la articulación de nuevos grupos sociales un potencial al menos implícitamente revolucionario, puesto que, conscientes y orgullosos 
de las conquistas que venían realizando tanto en el campo como en la ciudad, estos nuevos grupos - en el Perú, especialmente el cholo — se creían capaces de ampliar los avances que habían tenido. Eran capaces de proyectar un futuro que, aun no sabiendo la forma que tendría, no coincidía con el que se buscaba imponer desde los centros urbanos metropolitanos.

Sin embargo, las luchas sociales de izquierda en el espacio urbano son notoriamente débiles frente a las rurales. Quijano no nos brinda en este momento un análisis de ello, pero sí indica la necesidad de hacerlo —y lo hará, de forma copiosa, en la revista Sociedad y Política durante la década de 1970- Lo que sí nos brinda, es un extenso análisis de una cuestión que tenía en vilo a la teoría social latinoamericana, la dependentista en particular: la marginalidad. $\mathrm{Y}$ es posible leer, al menos a día de hoy, la marginalidad como una suerte de contraparte de la ausencia de un movimiento urbano fuerte. La clasificación social en Europa mediante el trabajo asalariado se dio a través de una homogeneización de la población alrededor de la industria en las ciudades. En América Latina, la industria y las ciudades más bien produjeron heterogeneidad, impidiendo o no dando la oportunidad de que las poblaciones urbanas o semiurbanas migrantes encontraran condiciones parejas para su nueva vida. De ahí que Quijano hablara de una estructuración parcial de la población marginal: eran efectivamente parte de la ciudad, siendo asimismo fundamentales para la lógica de dominación imperante, pero no tenían por ello una ubicación social consistente.

Pese a ello, desde que regresa de Chile a Perú, en 1971, Quijano dedicará la mayor parte de sus energías al fortalecimiento de los movimientos obreros, a quienes considerará entonces - de forma quizás sorpresiva para quienes leyeron sus textos hasta 1966 - como la clase convocada para liderar la lucha revolucionaria. El fracaso de esta alternativa llevó a que en 1983 Quijano cerrara Sociedad y Política, además de disolver la agrupación política que había formado con profesores, estudiantes y sindicalistas, el Movimiento Revolucionario Socialista.

A partir de los primeros años de la década de 1980, Quijano pasa a dudar de su apuesta política, pero no de la perversidad del enemigo. La derrota era dura, sobre todo por haber logrado que para la gran mayoría de las gentes se cerrara la posibilidad de cualquier horizonte alternativo (Quijano, 2001). La mera conciencia histórica, de capacidad de construcción del futuro, que había seducido a Quijano mediante Saint-Simon, se ponía en cuestión. 
A partir de entonces, los escritos de Quijano retoman cada vez más la pluralidad de dimensiones sociales que lo habían caracterizado en la década de 1960. Y, como en la década de 1950, son nuevamente los pueblos campesinos, ahora sobre todo bajo liderazgos propios y asumiendo la categoría indígena, las mayores fuerzas populares en contra del capital en su forma neoliberal. Si en algún momento de la década de 1970 había parecido que la urbanización latinoamericana lograría cierta homogeneización de su población, ello cae entonces por tierra y Quijano pasa a observar una "nueva heterogeneidad estructural en América Latina" (1989), la cual, asociada a sus relecturas sobre la modernidad en tanto acontecimiento global (1988), entre otras reflexiones, lo llevarán a considerar no solo la colonialidad del poder, sino la noción de heterogeneidad histórico-estructural (2014[2000]).

Los debates y los futuros quedaron, desde entonces, definitivamente reabiertos. 


\section{Bibliografía}

Assis Clímaco, D. (2014). Prólogo. En Quijano, A. Cuestiones y horizontes: de la independencia histórico-estructural a la colonialidad/descolonialidad del poder. Buenos Aires: CLACSO.

Bengoa, J. (2019). Aníbal Quijano. En The Clinic. Recuperado de https://www.theclinic. cl/2018/06/07/columna-jose-bengoa-anibal-quijano/.

Betancourt, M.(2013). Aníbal Quijano. Heterogeneidad histórico-estructural. Parte III [archivo de video]. Recuperado de https:/www.youtube.com/watch?v=NXrD1_gTbNQ.

Quijano, A. (2016). Prólogo. En Alba Herrera, C. A. Influencia del pensamiento de José Carlos Mariátegui en Huarás-Áncash. Huaraz: Waraq Quyllur.

Quijano, A. (2014[2000]). Colonialidad del poder y clasificación social. En Cuestiones y horizontes: de la independencia histórico-estructural a la colonialidad/descolonialidad del poder (pp. 285-327). Buenos Aires: CLACSO.

Quijano, A. (2001). El regreso del futuro y las cuestiones del conocimiento. En Hueso Húmero, 38, 3-18.

Quijano, A. (1989[1988]). Modernidad, identidad y utopía en América Latina. En Heinz,

R. S. (Comp.). ¿Nuevos temas, nuevos contenidos? Caracas: UNESCO, Nueva Sociedad.

Quijano, A. (1979a[1966]). Los movimientos campesinos contemporáneos de América Latina. Problema agrario y movimientos campesinos. Lima: Mosca Azul Editores.

Quijano, A. (1979b[1965]). El movimiento campesino peruano y sus líderes. En Problema agrario y movimientos campesinos. Lima: Mosca Azul Editores.

Quijano, A. (1980[1964]). Dominación y cultura. Lo cholo y el conflicto cultural en el Perú. Lima: Mosca Azul Editores.

Quijano, A. (1977a[1966]). Aspectos socioculturales de la urbanización en América Latina. En Dependencia, urbanización y cambio social en América Latina. Lima: Mosca Azul Editores.

Quijano, A. (1977b[1966]). Notas sobre el concepto de "marginalidad social". En Imperialismo y marginalidad en América Latina. Lima: Mosca Azul Editores.

Quijano, A. (1971a[1966]) [con el seudónimo de José Condoruna]. Las experiencias de la última etapa de las luchas revolucionarias en el Perú. En Bambirra, V. (Ed.). Diez años de insurrección en América Latina. Santiago de Chile: Ediciones Prensa Latinoamericana.

Quijano, A. (1971b[1965]). Imagen y tareas del sociólogo en la sociedad peruana. Preparando la convención de ciencias sociales. Lima: Pontificia Universidad Católica del Perú. 
Quijano, A. (1964a). La poesía: una praxis. Haraui, 1(2), 1-3.

Quijano, A. (1964b). Imagen saintsimoniana de la sociedad industrial. Revista de Sociología, 1(1), pp. 49-85. Universidad Nacional Mayor de San Marcos.

Quijano, A. (1962). C. Wright Mills, conciencia crítica de una sociedad de masas. En Revista del Museo Nacional, XXXI, 305-313. Lima.

Segato, R. (2015). La crítica de la colonialidad en ocho ensayos y una antropología por demanda. Buenos Aires: Prometeo. 